\title{
Chip Interleaved Multirate and Multimedia Transmission in Self-Encoded Communication System with Iterative Detection over Fading Channels
}

\author{
Liang Chi, Won-Mee Jang, Lim Nguyen \\ Department of Computer and Electronics Engineering, University of Nebraska-Lincoln, \\ Omaha, USA \\ E-mail: Ichi@unomaha.edu, \{wjang1,Inguyen1\}@unl.edu \\ Received July 26, 2011; revised August 26, 2011; accepted September 14, 2011
}

\begin{abstract}
In this paper, we investigate the self-encoded multirate and the multimedia (SEMM) transmission. In SEMM system, multiple applications transmit their information simultaneously with different bit rate via self-encoded spreading spectrum (SESS), where the spreading codes are derived from the previous bits rather than the pseudorandom code generator. At the transmitter, a block chip interleaving is employed to combat the deep fading over the wireless channels. At the receiver, a decorrelation scheme separates the combined signals to reduce the crosstalk between different applications, and provides a better estimation for the despreading sequence. In addition, interference cancelation (IC) is also adopted to improve both the correlation detection and iterative detection (ID) performance. The simulation results show that the proposed scheme significantly improves the performance over fading channels.
\end{abstract}

Keywords: Multirate, Multimedia, Decorrelation, Iterative Detection

\section{Introduction}

In previous work about the self-encoded multiple access (SEMA) system, every application transmits data with the same bit rate [1] and the chip interleaving SEMA with iterative detection achieves a significant performance improvement [2].

In SEMM transmission, each application employs SESS with different processing gain, where SESS is a time-varying spreading scheme which must update the despreading code with the detection bit. One challenge in SEMM system is to separate the combined signal at the receiver for each application. Another problem exists that the detection bit affected by multi-application interference is not accurate especially at lower SNR. In our paper, we employ a modified decorrelation scheme in which an extended signature vectors (ESV) is proposed for spreading signature [3] to overcome the two difficulties.

Iterative detection has been shown to improve the bit error rate (BER) performance for its time diversity [2]. The fundamental of the iterative detection is that the information bit exists in the next $\mathrm{N}$ spreading sequence consequently, where $\mathrm{N}$ is the chip length of the spreading spectrum. However, in SEMM system, the information bits are merged by the multi-application interference through the wireless channel. Therefore, an inference cancellation (IC) scheme is introduced to remove the multi application interference and realize the iterative detection.

The rest of this paper is organized as follows: in section 2, SEMM system model is introduced; in section 3, the numerical results are shown and in section 4, we make a conclusion for the scheme. The important notations in this paper are with following:

$l_{i}$ : the length of spreading sequences in application $i$;

$l_{\max }$ : the maximum length of all the spreading sequence;

$u$ : the total applications number in the SEMM system;

$m_{i}$ : the number of the spreading sequence of application $i$ in one transmission circle;

$\boldsymbol{p}_{i k}$ : the $k^{\text {th }}$ extend spreading sequence in application $i$;

$\boldsymbol{r}_{j}$ : the received vector from the $j^{\text {th }}$ row of the chip interleaver; 
$\boldsymbol{q}_{i}:$ the $i^{\text {th }}$ row of output of $\boldsymbol{Q}$;

$\boldsymbol{g}_{i k}$ : the $k^{\text {th }}$ despreading sequence in application $i$;

$\boldsymbol{G}$ : the extended signature vector of the SEMM system with size of $M \times l_{\max }$;

$\tilde{\boldsymbol{d}}$ : the correlation detection output;

$\hat{\boldsymbol{d}}:$ the decorrelation detection output.

\section{System Model}

\subsection{SEMM System}

Figure 1 shows the block diagram of SEMM transmission system. In the transmitter, each application employs the SESS as the code scheme where the spread spectrum is derived from the data source itself rather than the pseudorandom noise generator. The spreading chip from each application are combined together and sent to the chip interleaving blocks. The size of the block interleaving is $N \times N$ and $N=l_{\max }$ of all applications. At the receiver, deinterleaving is employed first, and then each application is correlated by its own despreading sequence with the crosstalk from other applications. To reduce this crosstalk, a decorrelation scheme is needed to separate the combined signal. Furthermore, interference cancelation removes the multiple application interference to realize the iterative detection. In SEMM system, to realize the decorrelation scheme, we propose an extended sig- nature vectors (ESV) to accommodate with the multirate transmission. The result of the decorrelation scheme plays an important role in SEMM system: on one side, the result updates the dispreading sequence for all applications; and on the other side, the interference cancelation still need the decorrelation result.

Figure 2 shows an example block diagram of SESS system with $N / T$ chip rate. The current transmission bit is spread by the output of a delay register which stores the previous $N$ bits, where $N$ is the longest sequence from all applications. As a result, the spreading sequence is not only randomly generated and independent from the current symbol, but also dynamically changing from one symbol to the next [1]. At the receiver, the recovered data provide an estimate of the transmitter code for despreading. The recovery data comes from the correlation detector definitely.

\subsection{Transmitter}

Figure 3 shows an example SEMM transmission with $u=3$ applications. From the diagram and our notation, in application 1, the number of spreading sequences is $m_{1}=4$ of length $l_{1}=4 \mathrm{chips} / \mathrm{bit} ;$ Application 2 is $m_{2}=2, l_{2}=8 \mathrm{chips} / \mathrm{bit}$; and Application 3 is $m_{3}=1$, $l_{3}=16 \mathrm{chips} / \mathrm{bit}$. Therefore, the total number of sequences in this time period is $M=m_{1}+m_{2}+m_{3}=7$.

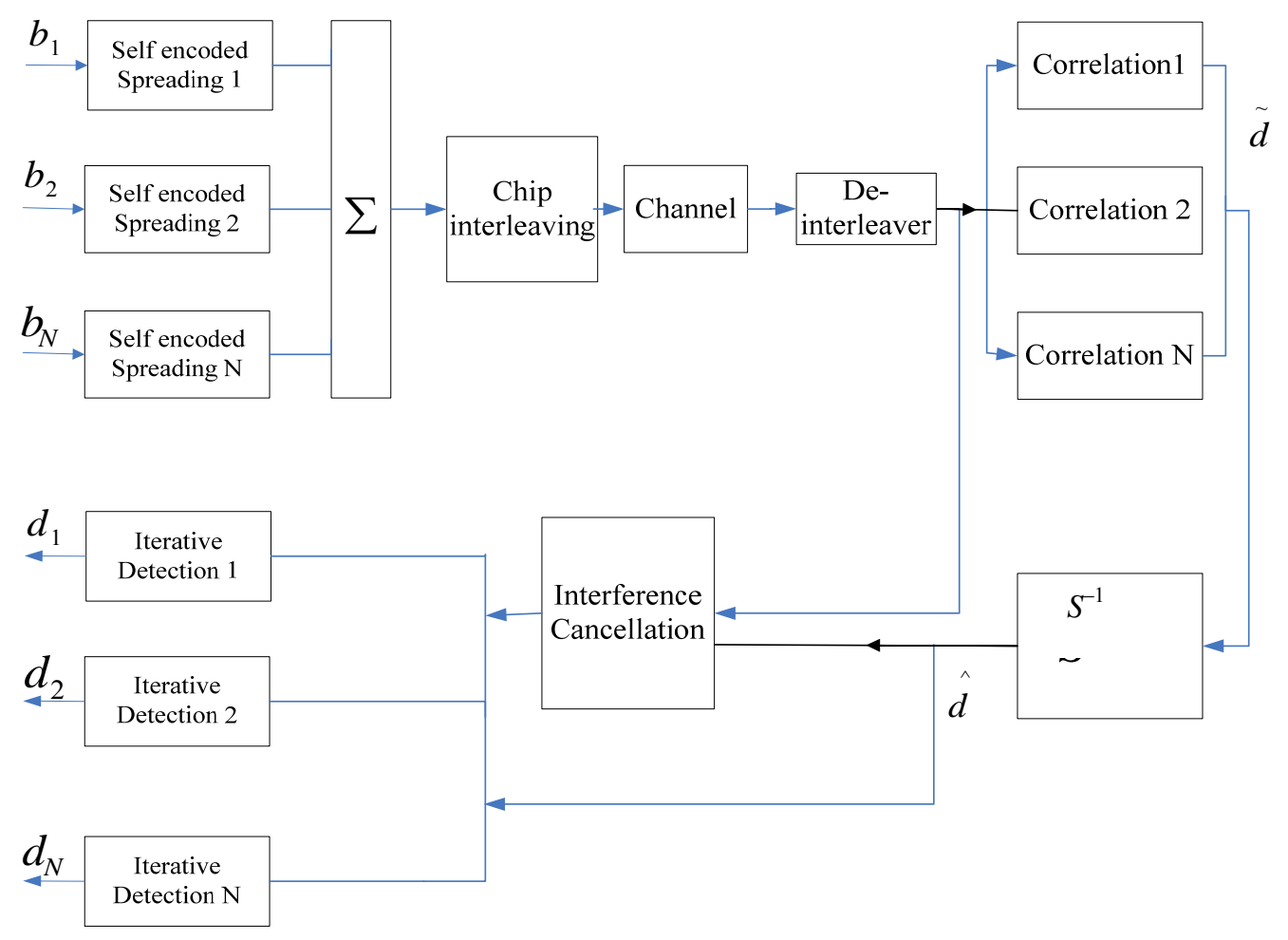

Figure 1. Block diagram of SEMM system. 


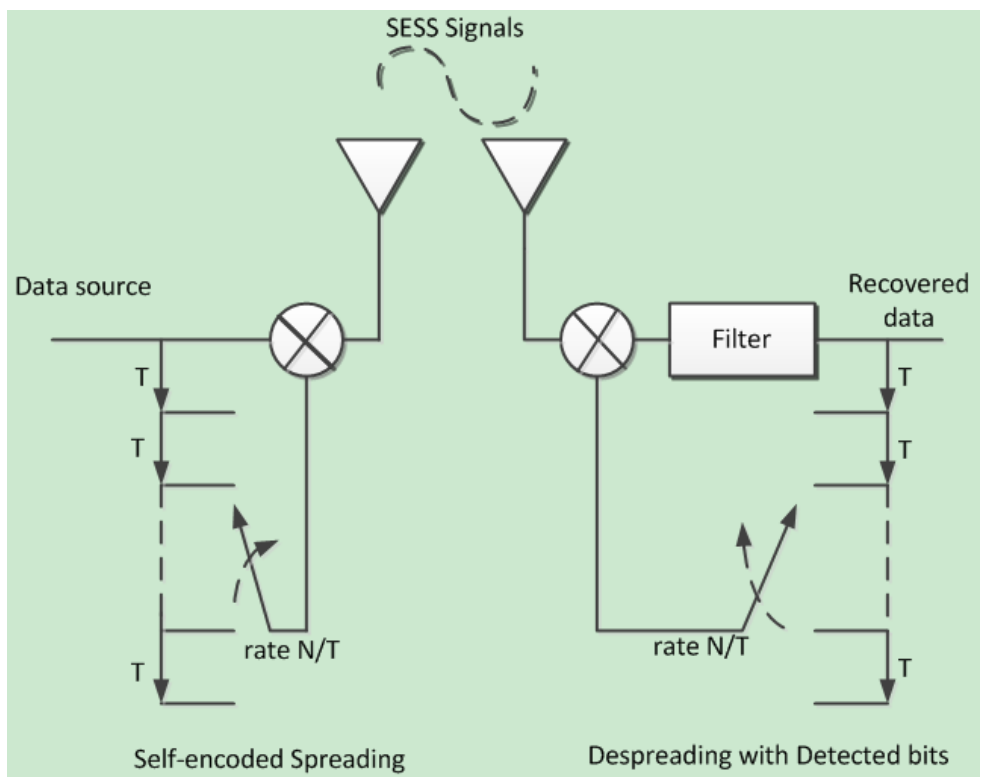

Figure 2. SESS communication system.

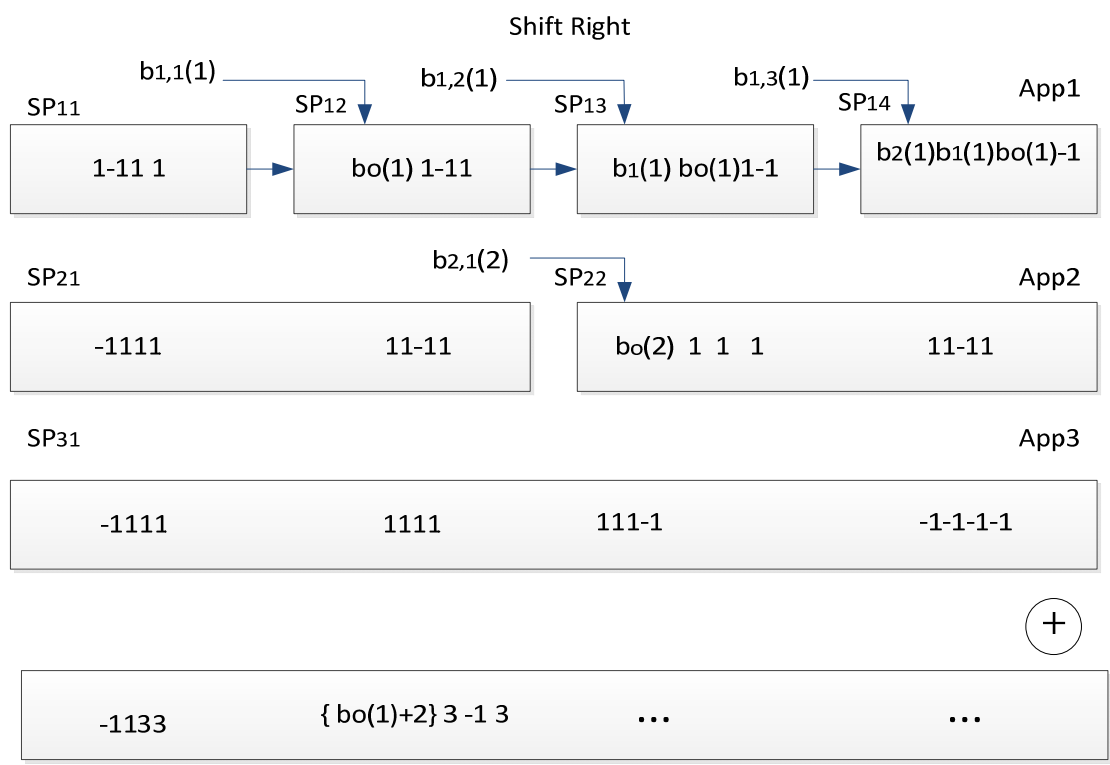

Figure 3. SESS multi-rate system transmission at the transmitter.

The spreading length of the system is $l_{\max }=l_{3}=16$. The $k^{\text {th }}$ spreading sequence of the $i^{\text {th }}$ application $\boldsymbol{S}_{i k}$ can be expressed as

$$
\boldsymbol{s}_{i k}=\left[b_{i k}(-1), b_{i k}(-2), \cdots, b_{i k}\left(-l_{i}\right)\right]
$$

where $b(-q)_{i k}$ is the previous $q^{\text {th }}$ transmitted bit of $k^{\text {th }}$ application.

This is used to encode the current bit $b_{i k}(0)$ as

$$
\boldsymbol{e}_{i k}=b_{i k}(0) \times \boldsymbol{s}_{i k}
$$

During one transmission period, then encoded sequence $\boldsymbol{e}_{i k}$ are extended to $l_{\max }$ by appending zeros at proper positions. The expanded sequence can be expressed as

$$
\boldsymbol{p}_{i k}=\left[\mathbf{0}_{(k-1) \times l_{i}} \boldsymbol{e}_{i k} \mathbf{0}_{l_{\max }-k \times l_{i}}\right]
$$

The encoded and expanded symbols from all applications are combined as

$$
\mathbf{z}=\Sigma_{i=1}^{M} \Sigma_{k=1}^{m_{i}} \boldsymbol{p}_{i k}
$$

where $\mathbf{z}$ is a $1 \times l_{\max }$ row vector as a input of the interleaver.

The combined signal undergoes block chip interleav- 
ing with fading is shown in Figure 4. We also assume that each column has the same fading, $\lambda_{i}$ where fading vector $\lambda$ is defined as

$$
\lambda=\left[\lambda_{1}, \lambda_{2}, \cdots, \lambda_{I_{\max }}\right]
$$

\subsection{Receiver}

From Figure 1, the signal from the channel will be assigned to the de-interleaver first. Therefore, the $k^{\text {th }}$ element of the $j^{\text {th }}$ signal vector $\boldsymbol{r}_{j}$ as the input of the interleaver can be expressed as

$$
r_{j}(k)=\boldsymbol{A}_{k k} \lambda z^{T}+n_{i}(k)
$$

where $k, j$ are from 1 to $N$; and $\boldsymbol{A}_{m n}$ is the amplifier of the $n^{\text {th }}$ output to $m^{\text {th }}$ input. The noise of $n_{i}(k)$ are following with AWGN distribution. The complete sequence of the $j^{\text {th }}$ block channel output can be given as

$$
\boldsymbol{r}_{j}=\left[r_{j}(1), r_{j}(2), \cdots, r_{j}(N)\right]
$$

where $j$ is from 1 to $N$ also. After the chip-interleaved sequences are received, we can reconstruct the original spreading sequence. The output of the de-interleaver is an $N \times N$ matrix which could be written as

$$
\boldsymbol{Q}=\left[\boldsymbol{r}_{1}^{T}, \boldsymbol{r}_{2}^{T}, \cdots, \boldsymbol{r}_{N}^{T}\right]
$$

We define the $i^{\text {th }}$ row of $\boldsymbol{Q}$ as $\boldsymbol{q}_{i}$

$$
\boldsymbol{q}_{i}=\left[r_{1}(i), r_{2}(i), \cdots, r_{N}(i)\right]
$$

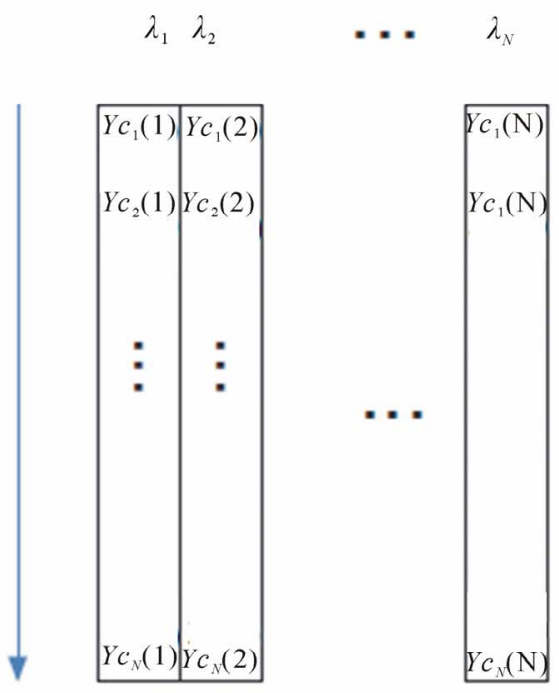

The correlation detection output $\tilde{\boldsymbol{d}}$ is an $M \times 1$ row vector and the element can be written as

$$
\tilde{\boldsymbol{d}}(k)=\boldsymbol{q}_{i} \times \boldsymbol{g}_{a b}^{T}
$$

where $k=1,2, \cdots, M$;

$i=1,2, \cdots, N ; a=1,2, \cdots, u ; b=1,2, \cdots, m_{a} ; \boldsymbol{g}_{a b}$ stands for the $b^{\text {th }}$ dispreading sequence for application $a$.

In the SEMM, the signature vectors $\boldsymbol{G}$ for decorrelation scheme is different from that of the multi-access system with same bit rate. The columns and rows must be extended for every application to accommodate with SEMM transmission.

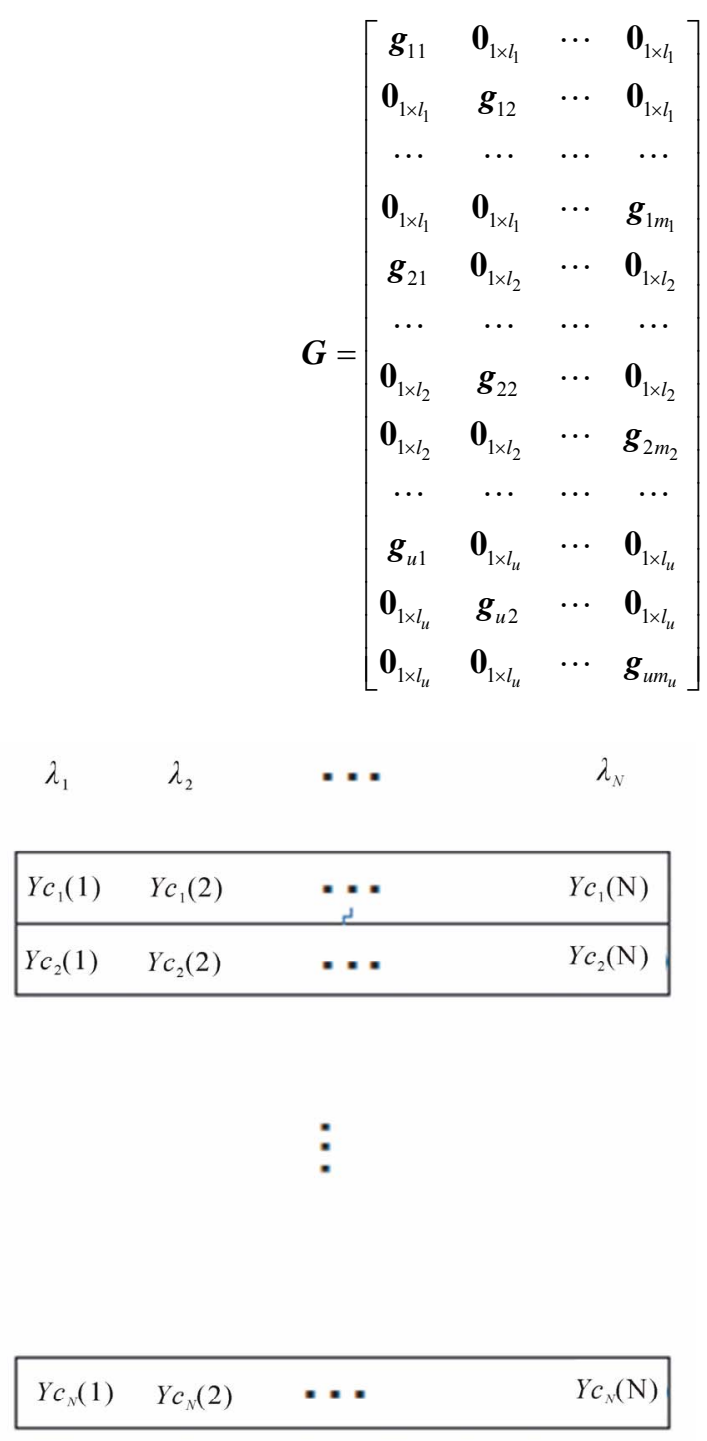

Figure 4. Block chip interleaving. 
In Equation (10), $\mathbf{0}_{1 \times k}$ stands for the $1 \times k$ row vector with elements of zero. The extended signature vectors can be used to generate the correlation matrix $\boldsymbol{S}$ [4-6]

$$
\boldsymbol{S}=\boldsymbol{G} \times \boldsymbol{G}^{T}
$$

and the output of the decorrelation scheme is

$$
\tilde{\boldsymbol{d}}=\boldsymbol{S}^{-1} \tilde{\boldsymbol{d}}
$$

We have mentioned that the ESV $\boldsymbol{G}$ plays an important role in interference cancellation part. In fact, we can devide the ESV $\boldsymbol{G}$ by row according to the application in SEMM system. It is clear that the first $m_{1}$ rows of $\boldsymbol{G}$ are composed by despreading sequence of Application-1; the next $m_{2}$ rows are composed by dispreading sequence of Application 2; and the last $m_{u}$ rows are composed by the despreading sequence of Application- $u$. So the sub-matrix $\boldsymbol{G}_{i}$ derived from $\boldsymbol{G}$ is

$$
\boldsymbol{G}_{i}=\boldsymbol{G}\left[(v+1):\left(v+m_{i}\right)\right]
$$

where

$$
v=\sum_{k=1}^{i-1} m_{i}
$$

$\boldsymbol{G}(m: n)$ means the sub-matrix begins from the $m$ th row of $S$ and terminates at the $n$th row of $\boldsymbol{G}$. So $G$ can be written as

$$
\boldsymbol{G}=\left[\begin{array}{c}
\boldsymbol{G}_{1} \\
\boldsymbol{G}_{2} \\
\vdots \\
\boldsymbol{G}_{u}
\end{array}\right]
$$

For interference cancellation, the remaining matrix $\breve{\boldsymbol{G}}_{k}$ and $\breve{\boldsymbol{d}}_{k}$ with fading are defined as

$$
\begin{gathered}
\breve{G}_{k}=\left[\begin{array}{c}
\boldsymbol{G}_{1} \\
\boldsymbol{G}_{2} \\
\vdots \\
\boldsymbol{G}_{k-1} \\
\boldsymbol{G}_{k+1} \\
\vdots \\
\boldsymbol{G}_{u}
\end{array}\right] \\
\breve{\boldsymbol{d}}_{k}=\left[\begin{array}{c}
\hat{d}_{1} \times \lambda_{1} \\
\hat{d}_{2} \times \lambda_{2} \\
\vdots \\
\hat{d}_{k-1} \times \lambda_{k-1} \\
\hat{d}_{k+1} \times \lambda_{k+1} \\
\vdots \\
\hat{d}_{u} \times \lambda_{u}
\end{array}\right]
\end{gathered}
$$

The interference cancelation result is

$$
\boldsymbol{C}=\boldsymbol{q}_{i}-\breve{\boldsymbol{G}}_{k} \breve{\boldsymbol{d}}_{k}
$$

From the discussion above, we find that the ESV plays the key role in the SEMM system which makes the decorrelation and interference cancelation available. After interference cancellation, the iterative detection can be employed as a single application [2,7].

\section{Simulation Result}

In the following, we present the performance of SEMM with three applications in additive white Gaussian noise (AWGN) and Rayleigh fading channels. The simulation result shows that the SEMM performance approaches single rate and is significantly better over the multirate random sequence. In this simulation, the SEMM employs three applications with chip length of $128 \mathrm{chips} / \mathrm{bit}, 64$ chips/bit and 32 chips/bit.

\subsection{AWGN Channel}

Figure 5 compares the SEMM system and single rate in AWGN channel. Notice that the SEMM performance approaches to the single rate. Taking application 1 with 128 chips/bit in SEMM as an example, at the BER of $10^{-3}$, SEMM is just $0.5 \mathrm{~dB}$ slightly worse than single rate. The performance different at higher SNR is primarily due to the multi-application interference.

In Figure 6, both SEMM and multirate random schemes are employed the decorrelation. However, iterative detection is applicable for SEMM only which is able to achieve about $3 \mathrm{~dB}$ gain over random sequence. That is because the iterative detection supplies a time diversity and double the receive energy to improve the BER performance.

\subsection{Flat Fading Channel}

In Figure 7 SEMM and single rate employ the iterative detection over Rayleigh fading channel. At the BER of $10^{-3}$, the SEMM is only at most $3 \mathrm{~dB}$ loss than the single rate. Still, the SEMM performance approaches to the single rate in Rayleigh fading channel.

Figure 8 shows the comparison of SEMM and multirate random sequence in the Rayleigh fading channel. Because iterative detection is only available for SEMM system, it achieves about $15 \mathrm{~dB}$ gain over random sequence at the BER of $10^{-3}$.

From all diagrams of the simulation result, we can make a conclusion that the performance of SEMM system works successfully in both AWGN and Rayleigh channel. With iterative detection scheme, the performance of the multirate system can approach to the single rate system. 


\section{Conclusions}

In this paper, we investigate the self-encoded multirate and the multimedia (SEMM) transmission. In SEMM system, the spreading sequence is time varied and updates by its own bit information. At transmitter side, chip interleaving is employed to combat the fading. At the receiver, we realize the decorrelation for multirate

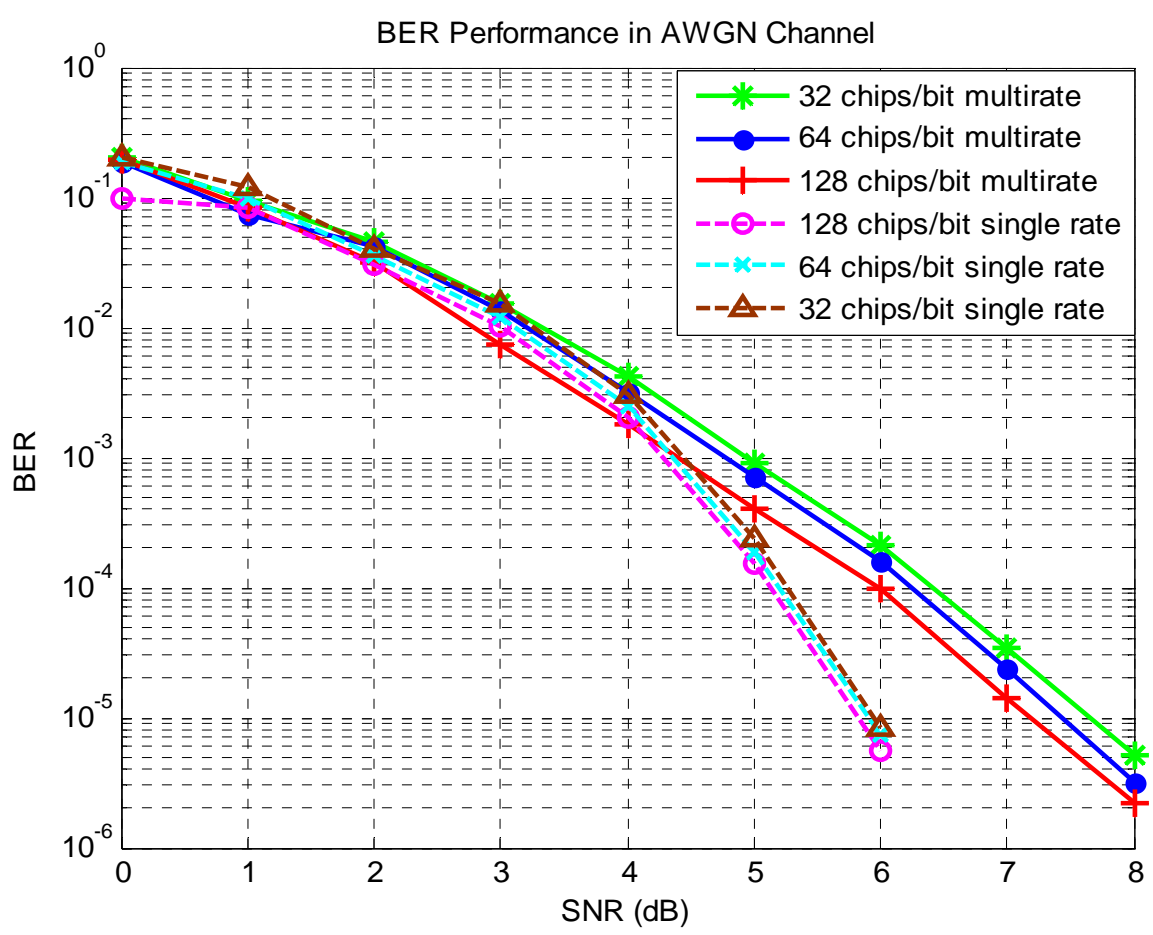

Figure 5. The performance of the SEMM in AWGN channel.

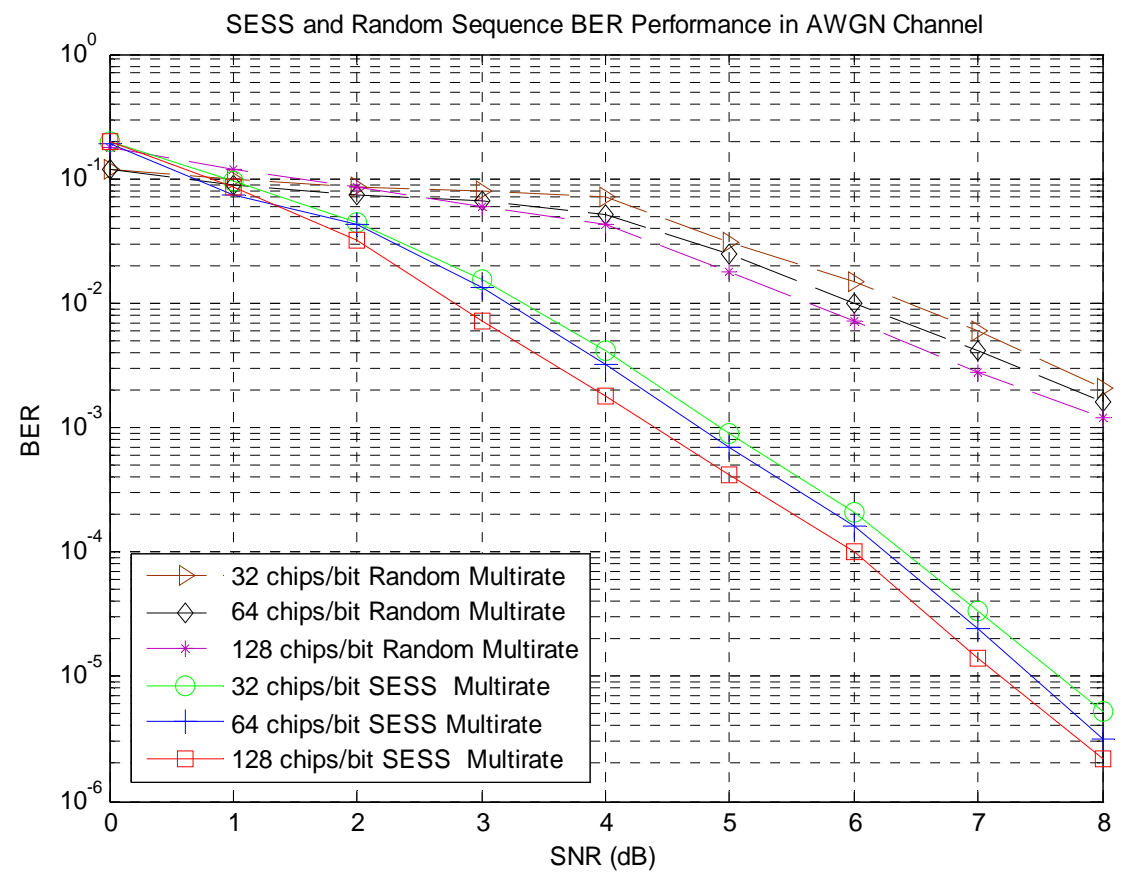

Figure 6. SEMM and random spreading sequence, multirate AWGN channel. 


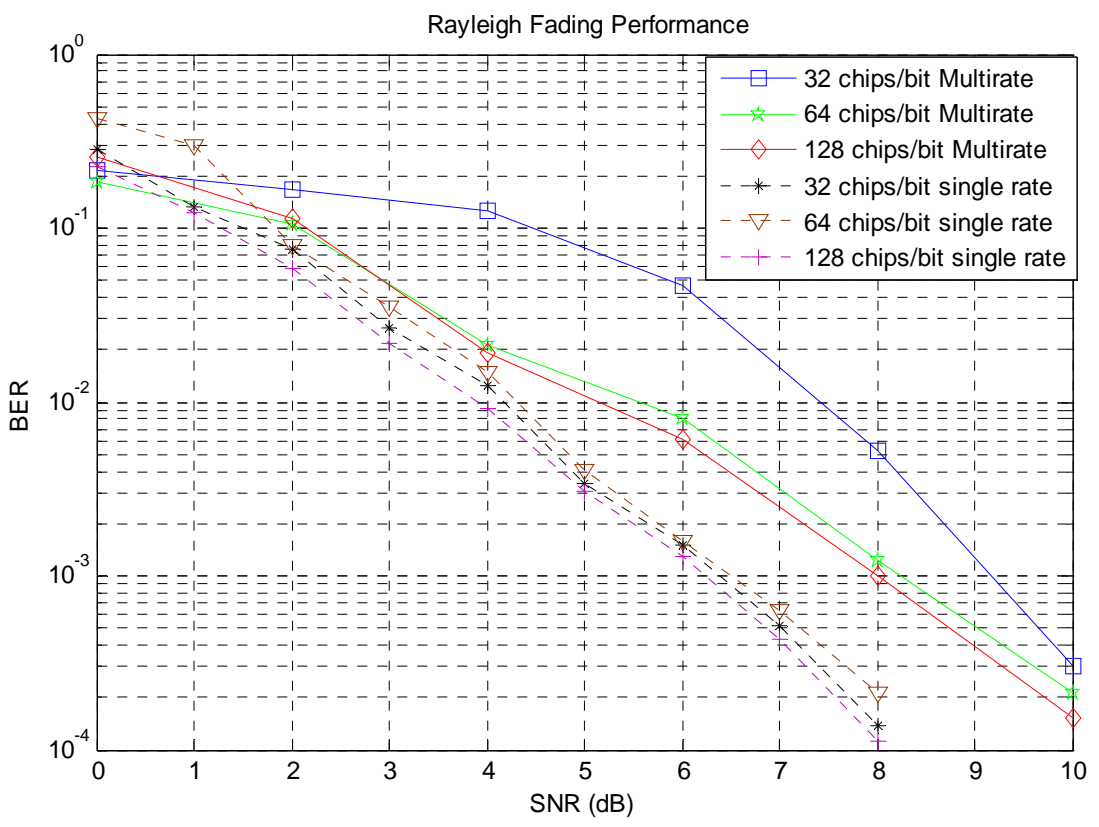

Figure 7. SEMM and single rate performance, rayleigh fading channel.

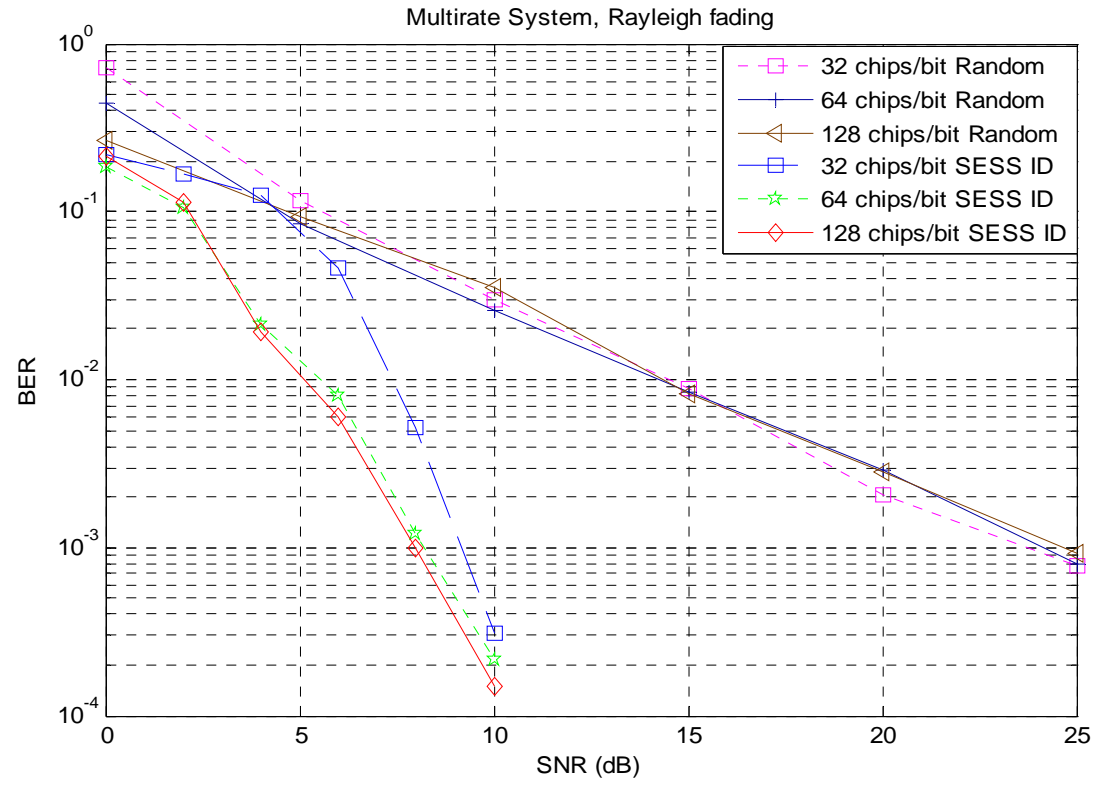

Figure 8. SEMM and random spreading sequence, multirate, rayleigh fading channel.

transmission. By the way, the decorrelation scheme does not depend on the code scheme and we still realize the multirate random sequence decorrelation in our simulation. Once from other applications, the crosstalk is removed the interference cancellation is employed to eliminate the multiapplication interference. Therefore, the iterative detection can be taken on as single rate. The simulation result shows that SEMM system with iterative detection works successfully in AWGN and Rayleigh channel. The performance approaches to the single rate and is significantly improved over multirate random sequence.

\section{Acknowledgements}

This work was supported by the contract award FA955008-1-0393 from the Air Force Office of Scientific Research. 


\section{References}

[1] L. Nguyen, "Self-Encoded Spread Spectrum and Multiple Access Communications," IEEE 6th International Symposium on Spread Spectrum Techniques and Applications, ISSSTA 2000, NJIT, New Jersey, Vol. 2, 6-8 September 2000, pp. 394-398.

[2] Y. S. Kim, W. M. Jang and L. Nguyen, "Chip-Interleaved Self-Encoded Multiple Access with Iterative Detection in Fading Channels," Journal of Communications and Networks, Vol. 9, No. 1, 2007, pp. 50-55.

[3] B. R. Vojcic and W. M. Jang, "Transmitter Precoding in Synchronous Multiuser Communications," IEEE Transactions on Communications, Vol. 46, No. 10, 1998, pp. 13461355. doi: $10.1109 / 26.725312$
[4] Sergio Verdu, "Multiuser Detection," Cambridge Press, Cambridge, 1998, pp. 234-244.

[5] W. M. Jang, L. Nguyen and M. Hempel, "Precoded Random Spreading Multiple Access System in AWGN Channels," IEEE Transactions on Wireless Communications, Vol. 3, No. 5, September 2004, pp. 1477-1480. doi:10.1109/TWC.2004.834686

[6] S. Verdu and S. Shamai, "Spectral Efficiency of CDMA with Random Spreading," IEEE Transactions on Information Theory, Vol. 45, No. 2, March 1999, pp. 622-640. doi:10.1109/18.749007

[7] L. Chi, W. M. Jang and L. Nguyen, "Self-Encoded Spread Spectrum for Multirate Multimedia Communication in Multi-Path Channel," 7th International Conference on Digital Content, Multimedia Technology and Its Applications, IDCTA2011, Busan, August 2011, pp. 16-18. 